\title{
Optimal rain rate estimation algorithm for light and heavy rain using polarimetric measurements
}

\author{
A. Elmzoughi ${ }^{1}$, R. Abdelfattah ${ }^{1,2}$, V. Santalla Del Rio ${ }^{3}$, and Z. Belhadj ${ }^{1}$ \\ ${ }^{1}$ URISA, Higher School of Communication of Tunis, University of Carthage, Tunisia \\ ${ }^{2}$ Département Image et Traitement de l'Information, Telecom Bretagne, France \\ ${ }^{3}$ EE de Telecomunicación, Universidad de Vigo, 36211, Vigo, Spain
}

Received: 18 November 2010 - Revised: 8 September 2011 - Accepted: 23 September 2011 - Published: 21 November 2011

\begin{abstract}
In this paper, we propose an ameliorated physically-based rain rate estimation algorithm for semi-arid regions using the Rayleigh approximation. The proposed algorithm simultaneously uses the reflectivity and the specific differential phase to provide an accurate estimation for both small and large rain rates. In order to validate the proposed estimator, simulated polarimetric rain rate data based on a dual approach, referring to both physical and statistical models of the rain target, are used. Moreover, experimental radar data (the same as used in Matrosov et al., 2006) taken in light to moderate stratiform rainfalls with rain rates varying between 2 and $15 \mathrm{~mm} \mathrm{~h}^{-1}$ were collected as part of the GPM pilot experiment. It is shown that the proposed algorithm for rain rate estimation based on the full set of polarimetric radar measurements agree better with in situ disdrometer ones.
\end{abstract}

\section{Introduction}

The rain rate is not a direct measurement of weather radars and it must be estimated from echoes of the rain events (Atlas, 1964). In the literature, many physically-based algorithms for rain quantification have been proposed (Atlas and Ulbrich, 1977, 1990). These algorithms are based in one or more measurable parameters as the reflectivity or the specific differential phase shift. Most algorithms lose accuracy either as the rain rate increases or as it decreases (Bringi and Chandrasekar, 2001; Seliga and Bringi, 1976). In semi-arid regions as the south of Spain or Tunisia more rain events are either very light or very strong, changing from some $\mathrm{mm} \mathrm{h}^{-1}$ up to $200 \mathrm{~mm} \mathrm{~h}^{-1}$.

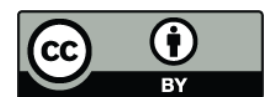

Correspondence to: R. Abdelfattah (riadh.abdelfattah@supcom.rnu.tn)
It is the purpose of this paper to develop an algorithm that presents good behavior on both extremes of the rain rate range for its application in semi-arid regions. S-band radars, that allow assuming Rayleigh scattering, will be considered. The final algorithm will be tested with simulated data. For the data simulations, a "real-like" weather data generator has also been developed that generates the data with the desired statistics from the physical properties of the rain drops.

The paper is organized as follows. The first part is dedicated to the theoretical design of the new rain rate estimation algorithm. Then the principle of the proposed "life-like" weather data generator is described. Finally, the new rain rate estimation algorithm is tested and validated using generated data.

\section{Theoretical considerations}

In this section we present the radar measurements used in polarization diversity radar for estimating rainfall rate with respect to the rain drops characteristics. Thus, we first describe widely used empirical models for the shapes, sizes, and velocities of rain drops. Then we present the reflectivity, the differential reflectivity, and the specific differential phase as well as their Rayleigh approximations.

\subsection{Rain drop characteristics}

The shapes of raindrops have been extensively studied theoretically (Green, 1975; Beard and Chuang, 1987) and experimentally in wind tunnels (Pruppacher and Beard, 1970), and in natural rainfall using aircraft probes (Chandrasekar et al., 1988; Bringi et al., 1998). The experimental results were fairly consistent with the theoretical results cited in the previous works. All of the above studies show that the shape of raindrops can be approximated by an oblate spheroid, described with an axis ratio $(b / a)$ and equivolumetric spherical

Published by Copernicus Publications on behalf of the European Geosciences Union. 
diameter $D$, where $a$ and $b$ are the major and the minor axes of the drop, respectively. A commonly used approximation relating the axis ratio of a raindrop to the diameter is given by Pruppacher and Beard in Pruppacher and Beard (1970):

$\frac{b}{a}=1.03-0.062 D$.

The drops sizes are characterized by their Drop Size Distribution (DSD), which represents the number of drops that have the same equivolume diameter $D$ in mm located in a volume of $1 \mathrm{~m}^{3}$ (Marshall and Palmer, 1948; Laws and Parsons, 1943). Ulbrich suggested the use of the gamma distribution for representing rain DSD in Ulbrich (1983):

$N(D)=N_{0} D^{\mu} \exp (-\Lambda D) \quad \mathrm{mm}^{-1} \mathrm{~m}^{-3}$.

The gamma DSD with three parameters $\left(N_{0}, \mu\right.$, and $\left.\Lambda\right)$ is capable of describing a broader range of raindrop size distributions than an exponential distribution. However, for reasons of unit normalization, a new form of the DSD was proposed during the design of the previous rain rate estimation algorithms:

$N(D)=N_{\mathrm{w}} f(\mu)\left(\frac{D}{D_{0}}\right)^{\mu} e^{\left(-\frac{(3.67+\mu) D}{D_{0}}\right)}$,

where $f(\mu)$ is a function that varies slowly with $\mu, D_{0}=$ $(3.67+\mu) / \Lambda$ is the median volume diameter in $\mathrm{mm}$, and $N_{\mathrm{w}}$ is given by:

$N_{\mathrm{w}}=N_{0} f(\mu)^{-1} D_{0}^{\mu} \quad \mathrm{mm}^{-1} \mathrm{~m}^{-3}$

The terminal velocity is the speed of the falling drop measured at sea level (Atlas et al., 1973). It depends not only on the raindrop diameter, but also on atmospheric pressure, humidity, and temperature. A useful experimental formula for terminal velocity proposed by Atlas and Ulbrich in Atlas and Ulbrich (1990) is:

$V(D)=3.778 D^{0.67} \mathrm{~ms}^{-1}$.

where $V$ designates the velocity and $D$ is always in $\mathrm{mm}$.

\subsection{Polarimetric weather radar measurements}

The reflectivity factor at horizontal and vertical polarizations noted, respectively, $Z_{\mathrm{hh}}$ and $Z_{\mathrm{vv}}$ can be expressed based on the drops backscattering amplitudes as (Bringi and Chandrasekar, 2001; Oguchi, 1983):

$Z_{\mathrm{hh}, \mathrm{vv}}=\frac{4 \lambda^{4}}{\pi^{4}|K|^{2}} \int\left|S_{\mathrm{hh}, \mathrm{vv}}(D)\right|^{2} N(D) d D \quad \mathrm{~mm}^{6} \mathrm{~m}^{-3}$,

where $\lambda$ is the radar wavelength, $K=\left(\varepsilon_{r}-1\right) /\left(\varepsilon_{r}+2\right)$ with $\varepsilon_{r}$ the water dielectric factor $S_{\mathrm{hh}}$ and $S_{\mathrm{vv}}$ are, respectively, the horizontal and vertical backscattering amplitudes. For an "Sband" $(\lambda=10 \mathrm{~cm})$ radar, rain drops, whose diameter ranges typically between a fraction of a millimeter and $5 \mathrm{~mm}$, act as Rayleigh scatterers. In this case, $\left|S_{\mathrm{hh}}\right|^{2}$ and $\left|S_{\mathrm{Vv}}\right|^{2}$ can be approximated as follows (Bringi and Chandrasekar, 2001):

$\left|S_{\mathrm{hh}, \mathrm{vv}}(D)\right|^{2}=C_{\mathrm{h}, \mathrm{v}} \frac{\pi^{4}|K|^{2}}{4 \lambda^{4}} D^{6}$,

where $C_{\mathrm{h}, \mathrm{v}}$ is a constant that depends on the polarization stat. Thus, using this approximation and evaluating the integral, the reflectivity $Z$ (refering either to $Z_{\mathrm{hh}}$ or $Z_{\mathrm{vv}}$ ) can be expressed as:

$Z=N_{\mathrm{w}} \times F_{Z}(\mu) \times D_{0}^{7} \quad \mathrm{~mm}^{6} \mathrm{~m}^{-3}$,

where $F_{Z}(\mu)$ is a function that varies slowly with $\mu$. Following Bringi and Chandrasekar (2001), the specific differential phase can be expressed as:

$K_{\mathrm{dp}}=\frac{180 \lambda}{\pi} \int \operatorname{Re}\left[f_{h}-f_{v}\right] N(D) d D \quad \operatorname{deg} \mathrm{Km}^{-1}$,

where $f_{\mathrm{h}}$ and $f_{\mathrm{v}}$ are the forward-scattering amplitudes at horizontal and vertical polarizations, respectively. $R e[]$ refers to the real part of a complex number. $f_{\mathrm{h}}$ and $f_{\mathrm{v}}$ can be analytically approximated in the Rayleigh domain (Bringi and Chandrasekar, 2001). Using these approximations, $K_{\mathrm{dp}}$ can be expressed as:

$K_{\mathrm{dp}}=\frac{0.0425}{\lambda} W D_{\mathrm{m}} \quad \operatorname{deg} \mathrm{Km}^{-1}$,

where $W$ is the rain water content defined as (the water volemic mass $\rho_{\mathrm{W}}$ was supposed to be equal to $1 \mathrm{~g} \mathrm{~cm}^{-3}$ and $\lambda$ is in $m)$ :

$W=\frac{\pi}{6} \int D^{3} N(D) d D \quad \mathrm{~g} \mathrm{~m}^{-1}$,

and $D_{\mathrm{m}}=(4+\mu) / \Lambda$ is the mass weighted diameter of the rain drops in $\mathrm{mm}$. The differential reflectivity is defined as the quotient of the horizontal and vertical reflectivities:

$Z_{\mathrm{dr}}=\frac{Z_{\mathrm{hh}}}{Z_{\mathrm{vv}}}$.

By evaluating $Z_{\mathrm{hh}}$ and $Z_{\mathrm{vv}}$, it is easy to prove that $Z_{\mathrm{dr}}$ is independent from $N_{\mathrm{w}}$ (Bringi and Chandrasekar, 2001). Thus, a microphysical link can be found between $Z_{\mathrm{dr}}, D_{0}$, and $D_{\mathrm{m}}$ since all of them depend only on $\mu$ and $\lambda$. Regressions made in Bringi and Chandrasekar (2001) show that:

$$
\begin{array}{rlrl}
D_{0} & = & 1.12 Z_{\mathrm{dr}}^{2.19} \\
& \text { and } \\
D_{\mathrm{m}} & =1.06 Z_{\mathrm{dr}}^{2.12}
\end{array}
$$

\subsection{The rain rate estimation}

In the still-air, the rain rate is given by Bringi and Chandrasekar (2001), Doviak and Zrnic (1993) as: 


$$
R=0.0018 \int v(D) D^{3} N(D) d D \quad \mathrm{~mm} \mathrm{~h}^{-1},
$$

where $v(D)$ is the terminal drop velocity in $\mathrm{m} \mathrm{s}^{-1}$ and $D$ in $\mathrm{mm}$. Replacing $v(D)$ by its approximation in Eq. (5) and evaluating the integral leads to the following relation:

$R=N_{\mathrm{w}} \times F_{R}(\mu) \times D_{0}^{4,67}$,

where $F_{R}(\mu)$ is a function that varies slowly with $\mu$. By doing the same with the water content defined in equation (11), we can also prove that:

$W=N_{\mathrm{w}} \times F_{\mathrm{w}}(\mu) \times D_{0}^{4}$,

where $F_{\mathrm{w}}(\mu)$ is a function that varies slowly with $\mu$. Starting from Eqs. (15) and (16), the rain rate and the water content can be related by the following expression:

$$
\frac{W}{N w}=\frac{F_{\mathrm{w}}(\mu)}{F_{R}(\mu)^{4 / 4.67}} \frac{R^{4 / 4.67}}{N_{\mathrm{w}}^{4 / 4.67}} .
$$

\section{The developed adapted rain rate estimation algorithm}

\subsection{Mathematical development}

Separate use of the reflectivity $Z$ or the specific differential phase $K_{\mathrm{dp}}$ leads to the design of rain rate estimation algorithm that provides accurate results for either light or heavy rain rates. This can be explained by the systematic errors in $Z$-based relations that lead to important estimation variabilities, especially for heavy rain rates and by the measurement errors in the $K_{\mathrm{dp}}$ estimation, which can not be accurately estimated for light rain rates (Brandes et al., 2001). Thus, in order to design a rain rate estimation algorithm adapted simultaneously to light and heavy rain rates, the reflectivity $Z$ and the specific differential phase $K_{\mathrm{dp}}$ must be used together. Several authors have already proposed algorithms that use several polarimetric parameters, see for examples Matrosov et al. (2002), Ryzhkov et al. (2005), Giangrande and Ryzhkov (2008).

Obtained algorithms are usually very sensitive to the measurements errors since these errors are amplified due to the large power coefficients of $Z, Z_{\mathrm{dr}}$, and $K_{\mathrm{dp}}$. In order to overcome this problem, a new variable has been introduced that expresses the degree of substitution of $D_{\mathrm{m}}$ or $D_{0}$ during the design of the algorithm. This led automatically to introduce $Z_{\mathrm{dr}}$ as a third input to the proposed algorithm in order to substitute the remained part of $D_{\mathrm{m}}$ or $D_{0}$. This new degree of freedom is then judiciously chosen so as to mitigate at maximum the effects of variabilities in $N_{\mathrm{w}}$ (which leads to minimization of systematic errors effects) and to obtain powerless algorithms (which leads to minimization of measurement errors effects). Exact mathematical development of the proposed estimation algorithm is presented in the following section.
In this work, the wavelength is fixed to $0.1 \mathrm{~m}$. However, general conclusions stay true for larger wavelengths since Rayleigh approximation is verified. Replacing $W$ in Eq. (10) by its expression in Eq. (17) and $\lambda$ by its value, $K_{\mathrm{dp}}$ can be expressed as:

$K_{\mathrm{dp}}=0.425 \frac{F_{\mathrm{w}}(\mu)}{F_{R}(\mu)^{4 / 4.67}} N_{\mathrm{w}}^{0.67 / 4.67} R^{4 / 4.67} D_{\mathrm{m}} \quad \operatorname{deg} \mathrm{Km}^{-1}$

The conventional $K_{\mathrm{dp}}$-based rain rate estimation algorithms rise directly from the above relation. On the other hand, by combining Eqs. (8) and (15), it can be proved that:

$Z=\frac{F_{Z}(\mu)}{F_{R}(\mu)} \times R \times D_{0}^{2.33}$.

Remembering that the median volume diameter $D_{0}=$ $(3.67+\mu) / \Lambda$ and that the mass weighted diameter of the rain drops $D_{\mathrm{m}}=(4+\mu) / \Lambda$, it can be easily inferred that $D_{0}$ and $D_{\mathrm{m}}$ are very close. Regressions made on real data in Bringi and Chandrasekar (2001) show that:

$D_{0}=0.95 \times D_{\mathrm{m}}$.

Thus, Eq. (19) can be rewritten as:

$Z \approx 0.88 \times \frac{F_{Z}(\mu)}{F_{R}(\mu)} \times R \times D_{\mathrm{m}}^{2.33}$.

Now a new degree of freedom $x$ is introduced in the power of the mass weighted diameter $D_{\mathrm{m}}$. Thus, Eqs. (18) and (21) can be respectively expressed as follows:

$K_{\mathrm{dp}}^{x}=0.425^{x}\left(\frac{F_{\mathrm{w}}}{F_{R}(\mu)^{4 / 4.67}}\right)^{x} N_{\mathrm{w}}^{0.67 x / 4.67} R^{4 x / 4.67} D_{\mathrm{m}}^{x}$.

and

$Z=0.88 \frac{F_{Z}(\mu)}{F_{R}(\mu)} R D_{\mathrm{m}}^{2.33-x} D_{\mathrm{m}}^{x}$.

Finally, by substituting $D_{\mathrm{m}}$ in Eq. (22) using Eq. (23), a new $x$-parameterized relation can be designed:

$$
\begin{aligned}
R= & {\left[\frac{0.88 F_{z}}{F_{R}}\left(\frac{0.425 F_{\mathrm{w}}}{F_{R}^{\frac{4.67}{4.67}}}\right)^{-x}\right]^{\frac{4.67}{4 x-4.67}} } \\
& \times N_{\mathrm{w}}^{\frac{0.67-67 x}{4.67 x}} \times Z^{\frac{4.67}{4.67-4 x}} \times K_{\mathrm{dp}}^{\frac{-4.67-4 x}{4.67 x}} \times D_{\mathrm{m}}^{\frac{4.67(2.33-x)}{4 x-4.67}} .
\end{aligned}
$$

Considering the fact that both $D_{\mathrm{m}}$ and $Z_{\mathrm{dr}}$ depend on the same DSD parameters in the Rayleigh approximation, a simple relation can be obtained between these two parameters. namely:

$D_{\mathrm{m}}=a Z_{\mathrm{dr}}^{b}$,

where the coefficients $a$ and $b$ depend on the region and season and must be estimated from a measurement database. 
Using the above relation, Eq. (24) can be transformed to the following $x$-parameterized relation:

$$
\begin{aligned}
R= & {\left[\frac{0.88 a F_{z}}{F_{R}}\left(\frac{0.425 F_{\mathrm{w}}}{F_{R}^{\frac{4.67}{4.67}}}\right)^{-x}\right]^{\frac{4.67}{4 x-4.67}} } \\
& \times N_{\mathrm{w}}^{\frac{0.67-4 x}{4.67-4 x}} \times Z^{\frac{4.67}{4.67-4 x}} \times K_{\mathrm{dp}}^{\frac{-4.67 x}{4.67-4 x}} \times Z_{\mathrm{dr}}^{\frac{4.67 b(2.33-x)}{4 x-4.67}} .
\end{aligned}
$$

Finally, if the power of $N_{\mathrm{w}}$ is near to zero, the above result can be further refined by a family of $R\left(Z, K_{\mathrm{dp}}, Z_{\mathrm{dr}}\right)(x)$ estimators of the rain rate of the form:

$$
R=a(x) Z^{b(x)} K_{\mathrm{dp}}^{c(x)} Z_{\mathrm{dr}}^{d(x)} .
$$

Now, it will be of interest to look for a value of $x$ that not only guaranties an absolute value of zero near power in $N_{\mathrm{w}}$, but also powerless coefficients in $Z$ and $K_{\mathrm{dp}}$ in order to mitigate the total error effects.

\subsection{Optimization of the proposed algorithm}

Given that the mitigation of the total error of the proposed algorithm is possible through the simultaneous minimization of power coefficients' modules of $N_{\mathrm{w}}, Z$ and $K_{\mathrm{dp}}$. This problem can be considered as a minimum square minimization problem and it is equivalent to minimize the sum of the square of power coefficients of these measurements. So, a minimization criterion $J$ can be designed as follows:

$J=\left(\frac{0.67 x}{4.67-4 x}\right)^{2}+\left(\frac{4.67}{4.67-4 x}\right)^{2}+\left(\frac{-4.67 x}{4.67-4 x}\right)^{2}$

Study of the minimization criterion shows that it presents an absolute minimum for $x \approx-0.9$ as shown in Fig. 1. Evaluating power coefficients for $x=-0.9$ leads to a power of -0.072 for $N_{\mathrm{w}}$ term, a power of 0.56 for $Z$ and a power of 0.50 for $K_{\mathrm{dp}}$. It is clear that the power coefficient of $N_{\mathrm{w}}$ is close to zero. Also, powers coefficients of $Z$ and $K_{\mathrm{dp}}$ are less than the unity, which traduce a powerless relation between $R, Z$, and $K_{\mathrm{dp}}$. Consequently, use of the found value of $x$ leads to minimize both systematic error effects (through minimizing $N_{\mathrm{w}}$ power coefficient) and measurement error effects (through minimizing the $Z$ and $K_{\text {dp }}$ power coefficients).

\section{The proposed real-like polarimetric weather radar measurements generator}

In this section a "real-like" weather radar data generator using the Rayleigh approximation is developed.

\subsection{General description of the proposed simulator}

The proposed simulator is based on a three step approach (Ryzhkov et al., 2005). The first step consists of adopting a physical model of the rain drops which takes into account not only the Drop Size Distribution (DSD) presented in Sect. 2.1, but also the Drop Orientation Distribution. In the second

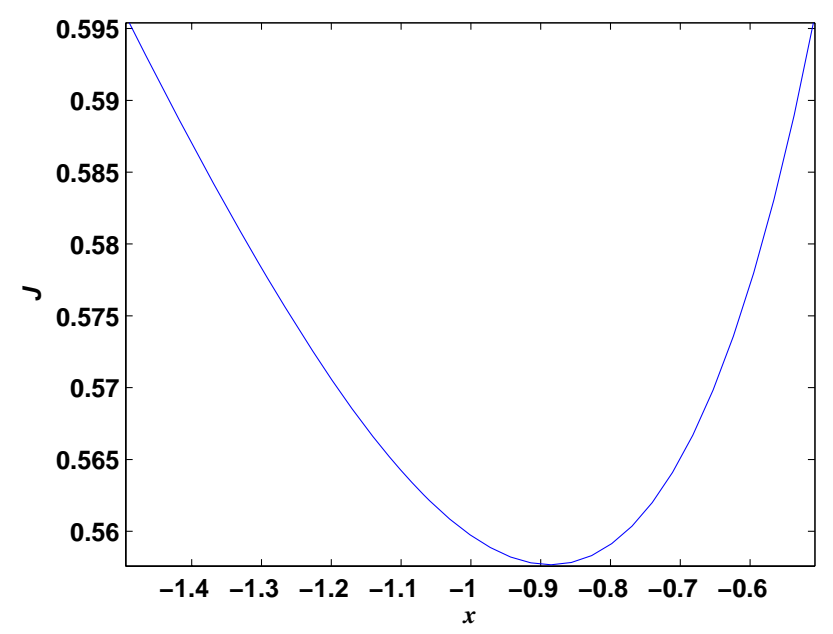

Fig. 1. Optimization criterion.

level, we adopt a statistical model that consider the radar rain returns as realizations of a multivariate Gaussian random variable. Finally, the outputs of the physical model are used as inputs of the statistical one according to the Ergodicity principle in order to generate the "real-like" data. The proposed simulator is highly modular, allowing the emulation of a wide variety of the rain physical processes thanks to its great flexibility in fixing the starting physical properties of the rain drops and the geometrical configuration of the radar. Also, it is envisageable to implement additional modules in the simulator, such as the Mie and the $T$-Matrix approximations and the Doppler radial winds modules. An extension of the proposed simulator to the 2-D case is published in Elmzoughi et al. (2007).

\subsection{The physical model}

Besides the shape, the size distribution, and the velocity of the rain drops presented in Sect. 1, the considered physical model describes the geometrical configuration of the rain drop when the spheroid symmetry axis is oriented along $O N$ with angles $\theta_{\mathrm{b}}$ and $\phi_{\mathrm{b}}$. The angle between the incident direction $O I$ and $O N$ is $\psi . Q T$ is the projection of $O N$ onto the plane of polarization of the incident wave. $Q V$ is the projection of $O Z$ onto the plane of polarization of the incident wave. The canting angle is the angle measured clockwise between the line segments $Q V$ and $Q T, \beta=\widehat{V Q T}$.

The orientation of the symmetry axis of a particle can be completely described by the couple ( $\psi$ (relevant for scattering), $\beta$ (relevant for canting)) since they are not relative to the choice of the XYZ system. Several authors, notably McCormick and Hendry (McCormick and Hendry, 1979), have assumed that the PDF (Probability Density Function) of $\beta$ is a symmetric distribution. Oguchi has assumed that $\beta$ and $\psi$ are normal distributed and that $\beta, \psi$ and $D$ are independent (Oguchi, 1983). Here, Oguchi's model has been adopted. 


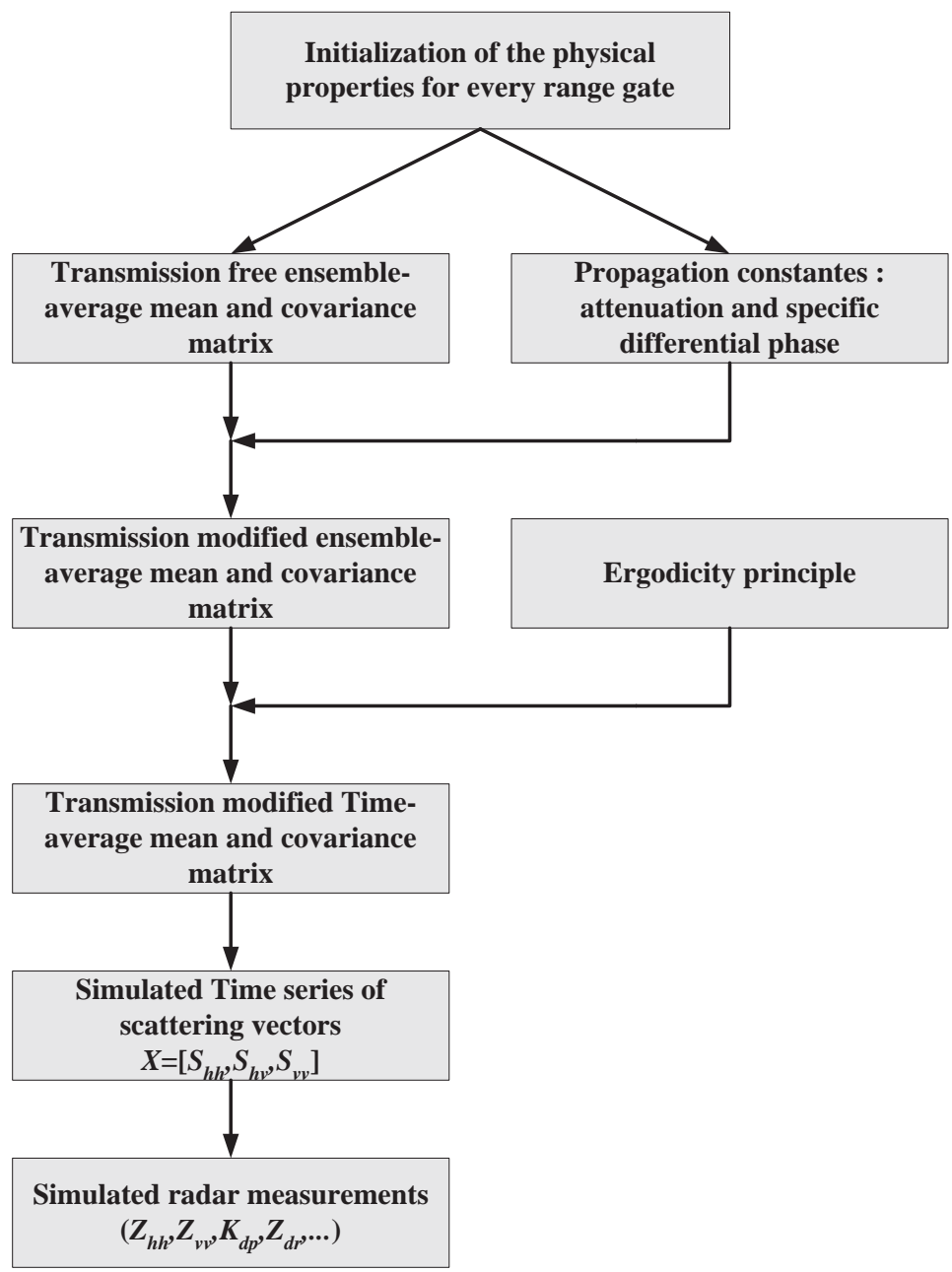

Fig. 2. The block diagram for the principle of the proposed data generator.

\subsection{The statistical model}

It is widely accepted by the meteorological community (Bringi and Chandrasekar, 2001) that the scattering vector $X=\left(S_{\mathrm{hh}}, S_{\mathrm{hv}}, S_{\mathrm{vv}}\right), X$ can be modelled as having a multivariate complex Gaussian distribution:

$P_{X}(x)=\frac{1}{\pi^{3}|C|} e^{-x^{T} C^{-1} x}$,

where $C$ is the complex covariance matrix, $T$ indicates the complex conjugate, and $|\cdot|$ is the determinant.

\subsection{Ergodicity principle and polarimetric data generation}

It was shown in Bringi and Chandrasekar (2001) and Tough et al. (1995) that the radar returns from a distributed target verify the ergodicity property. Thus, the time-average is equal to the ensemble average of the radar returns, and the time covariance matrix can be expressed as an ensemble-average covariance matrix. So, considering the physical model described below, time-statistics can be obtained by averaging over both the DSD and the orientation distribution. From an electromagnetic point of view (Guifu et al., 2001), the scattering amplitudes of a fixed rain drop whose orientation is described by the couple $(\psi, \beta)$ verify the following relations:

$$
\begin{aligned}
& S_{\mathrm{hh}}=A f_{\mathrm{a}}+B f_{\mathrm{b}} \\
& S_{\mathrm{hv}}=E\left(f_{\mathrm{a}}-f_{\mathrm{b}}\right), \\
& S_{\mathrm{vv}}=C f_{\mathrm{a}}+D f_{\mathrm{b}}
\end{aligned}
$$

where $A, B, C, D$, and $E$ depend only on the orientation $(\psi, \beta)$ of the drop, and $f_{\mathrm{a}}$ and $f_{\mathrm{b}}$ are the complex backscattering amplitudes corresponding respectively to the horizontal and vertical polarization in the principal plane $(\perp$ to the propagation direction). They depend on the microphysical properties of the drop, namely the size and the dielectrical constant of the water. In the Rayleigh approximation, they can be 


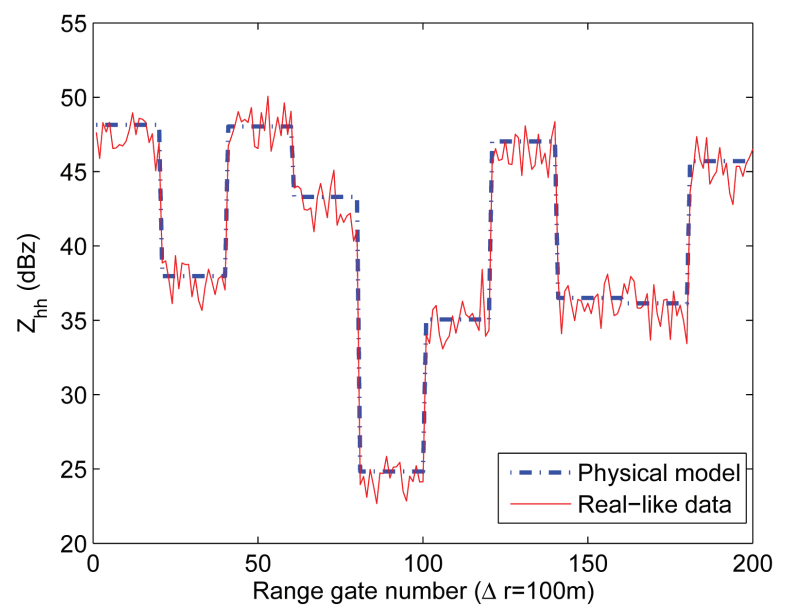

(a)

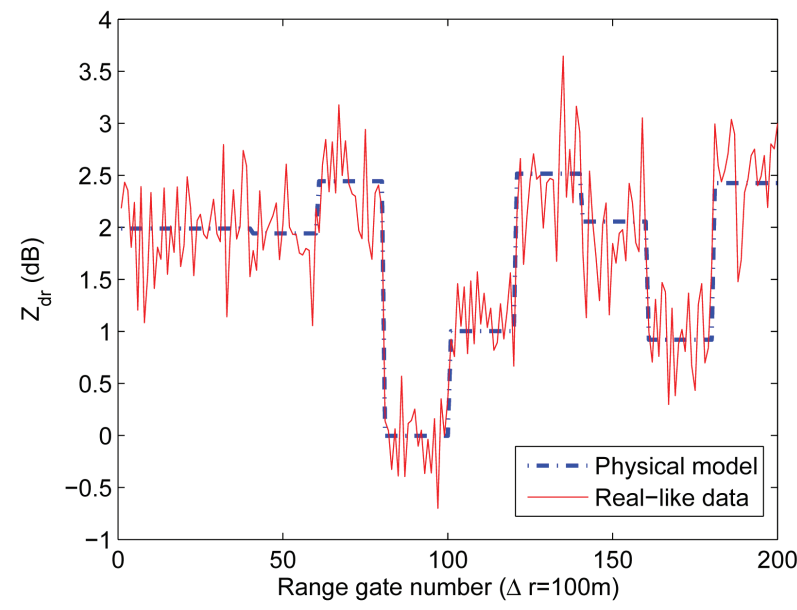

(c)

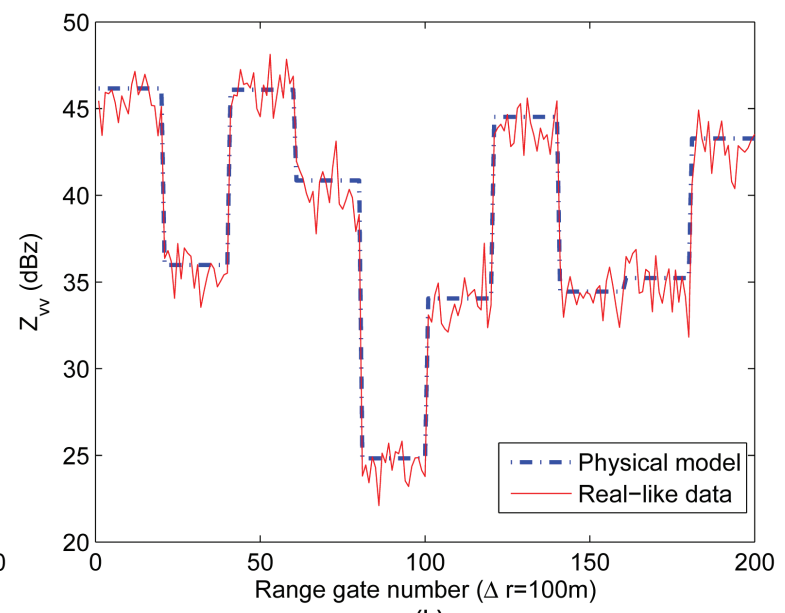

(b)

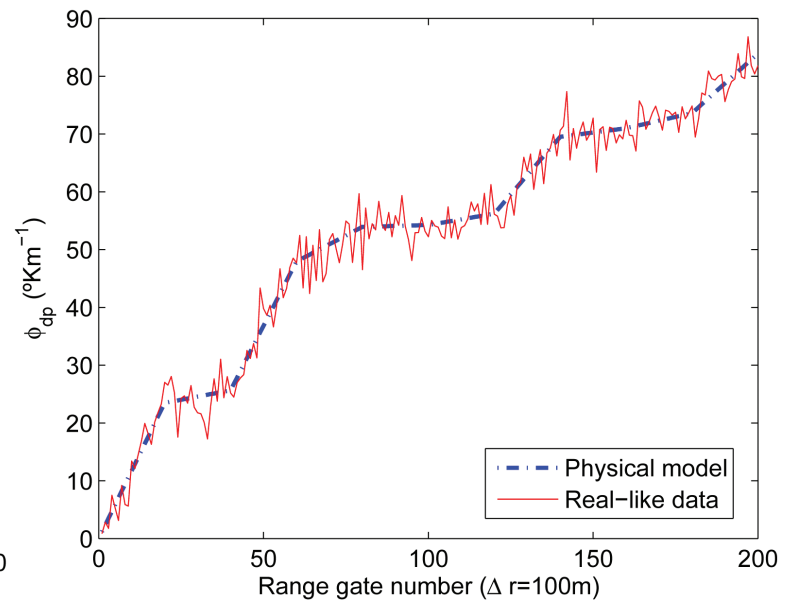

(d)

Fig. 3. Example of the generated data (a) horizontal reflectivity (b) vertical reflectivity (c) the differential reflectivity (d) differential phase shift.

analytically computed. Moreover, they can be approximated as

$$
\begin{aligned}
& f_{\mathrm{a}}=\alpha_{\mathrm{a}} D^{\beta_{\mathrm{a}}} \\
& f_{\mathrm{b}}=\alpha_{\mathrm{b}} D^{\beta_{\mathrm{b}}},
\end{aligned}
$$

where $\alpha_{\mathrm{a}}, \beta_{\mathrm{a}}, \alpha_{\mathrm{b}}$, and $\beta_{\mathrm{b}}$ are adjusted following the water activity (stimulating radar wavelength and water temperature), and $D$ is the drop diameter. Now, let $<X>=\left(<S_{\mathrm{hh}}>,<\right.$ $S_{\mathrm{hv}}>,<S_{\mathrm{vv}}>$ ) be the average scattering vector. Given that $\psi, \beta$ and $D$ are independent, it is easy to prove that:

$$
\begin{aligned}
& <X>=\left(\bar{A}<f_{\mathrm{a}}>+\bar{B}<f_{\mathrm{b}}>, \bar{E}<f_{\mathrm{a}}-f_{\mathrm{b}}>,\right. \\
& \left.\bar{C}<f_{\mathrm{a}}>+\bar{D}<f_{\mathrm{b}}>\right)
\end{aligned}
$$

where the upperline designates the averaging over orientation and $<>$ is averaging over the (DSD). In order to take into account the propagation effects, propagation constants $\gamma_{\mathrm{h}}$ and $\gamma_{\mathrm{v}}$ can be analytically computed using the Oguchi solution presented in Oguchi (1983). Finally,

$<X>=\left(<S_{\mathrm{hh}}>e^{-2 \gamma_{\mathrm{h}} r},<S_{\mathrm{hv}}>e^{-\left(\gamma_{\mathrm{h}}+\gamma_{\mathrm{v}}\right) r},<S_{\mathrm{Vv}}>e^{-2 \gamma_{\mathrm{v}} r}\right)$
Elements of the ensemble-average covariance matrix $C$ can be computed in the same way. Once physical statistics are computed, radar returns are generated as a limited number of realizations of a multivariate Gaussian variable following the statistical model and according to the ergodicity principle. Then the covariance matrix is estimated from the generated scattering vector. From the estimated covariance matrix the principal radar measurements namely the horizontal and vertical reflectivities $Z_{\mathrm{hh}}$ and $Z_{\mathrm{vv}}$, the differential reflectivity $Z_{\mathrm{dr}}$ and the differential phase shift $\phi_{\mathrm{dp}}$ from which is estimated $K_{\mathrm{dp}}$ are estimated. Diagram in Fig. 2 summarizes the principle of the proposed data generator.

\section{Simulation and result analysis}

The simulation process consists of three parts. The first one is dedicated to test and validate the proposed data generator. In the second part, different physically-based rain rate 
Table 1. The considered variability ranges for the drop size.

\begin{tabular}{ccc}
\hline Parameter & Range of variability & Unit \\
\hline$N_{\mathrm{w}}$ & {$\left[10^{3}, 10^{5}\right]$} & $\mathrm{mm}^{-1} \mathrm{~m}^{-3}$ \\
$D_{0}$ & {$[0.5,2.5]$} & $\mathrm{mm}$ \\
$\mu$ & {$[-1,5]$} & (none) \\
\hline
\end{tabular}

estimators are compared. Finally, a rigorous error structure analysis is performed. A dimilar simulation and result analysis with a different approch is given in (Giangrande and Ryzhkov, 2008).

\subsection{Validation of the proposed "real-like" polarimetric data generator}

A range profile consisting of 200 range gates of depth $\Delta r=$ $100 \mathrm{~m}$ has been generated. Homogeneity has been assumed for 20 consecutive gates (by invoking the ergodicity principle, the time mean and covariance matrix, of vector $\boldsymbol{X}$, are equal for each range gate and could be evaluated as done in the previous section given the physical properties of the drops).

The wavelength was fixed to $10 \mathrm{~cm}$; the temperature to $10^{\circ} \mathrm{C}$. The distribution of $\psi$ was $\mathcal{N}\left(90^{\circ}, 4^{\circ}\right)$ and the distribution of $\beta$ was $\mathcal{N}\left(0^{\circ}, 4^{\circ}\right)$. The DSD parameters $N_{\mathrm{w}}, D_{0}$, and $\mu$ are fixed independently in the ranges presented in Table 1 (Ulbrich, 1983), allowing rain rates between 0 and $200 \mathrm{~mm} \mathrm{~h}^{-1}$. However, we had taken into account only usual rainfall rates in semi-arid regions. The number of samples for every range gate is 16 . Starting from these physical parameters, "real-like" radar returns were generated from which the "real-like" $Z_{\mathrm{hh}}, Z_{\mathrm{vv}}, Z_{\mathrm{dr}}$, and $\phi_{\mathrm{dp}}$ were deduced. Figure 3 shows the obtained results.

Figure $3 \mathrm{a}, \mathrm{b}, \mathrm{c}$, and d represent, respectively, the horizontal reflectivity $Z_{\mathrm{hh}}$, the vertical one $Z_{\mathrm{vv}}$, the differential reflectivity $Z_{\mathrm{dr}}$, and the differential phase shift $\phi_{\mathrm{dp}}$ obtained using the proposed simulator. These figures show that the real measurements appear as the sum of the exact physical measurements and a random noise. This noise results from the fact that the rain is a time-varying and distributed target. Indeed, we can verify that the real-like reflectivities range between 0 and $60 \mathrm{dBz}$ and the differential reflectivity ranges between -1 and $4 \mathrm{~dB}$, which are very well known intervals for the rain target (Bringi and Chandrasekar, 2001). Also, the reflectivities are highly correlated in accordance with results in the literature, which indicates that the correlation factor for rain targets is over 0.97. Finally, it is important to remark that the specific differential phase $K_{\mathrm{dp}}$, which is obtained as the slope of a local linear regression made on the differential phase $\phi_{\mathrm{dp}}$, will be very noisy for small values due to the noise resulting from the scattering differential phase. To avoid this problem, a smooth version of $\phi_{\mathrm{dp}}$ is used to estimate $K_{\mathrm{dp}}$.
Consequently, results obtained by the proposed simulator behave as real measurements and can be used as a laboratory realistic tool to validate and compare rain rate estimation algorithms. In order to keep an acceptable spatial resolution and to guarantee a good denoising of $\phi_{\mathrm{dp}}$, the smoothing is performed by averaging along every 5 consecutive range gates.

\subsection{Rain rate estimation physically-based algorithms comparison}

In order to evaluate the performance of the adopted technique, the $R(Z), R\left(Z, Z_{\mathrm{dr}}\right), R\left(K_{\mathrm{dp}}\right)$, and $R\left(K_{\mathrm{dp}}, Z_{\mathrm{dr}}\right)$ algorithms have been considered for comparison. These algorithms follow a power law relation as shown in Table 2 (coefficients were computed by performing non linear regressions). These results are roughly similar to those obtained for X-band radar by Matrosov et al. (2002). Then corresponding coefficients can be estimated by performing non linear regressions. The least square algorithm described in Marquardt (1963) is used for that. In order to ensure good fittings, initialization of the regression algorithm is very important. Multiplicative coefficients $a$, which depend on both $N_{\mathrm{w}}$, and $\mu$ for all the algorithms, were initialized by averaging over these two parameters. The power coefficients namely $b, c$, and $d$-were initialized to their theoretical values. Simulated data is now used as input to the least square non linear algorithm. Table 2 summarizes results for fitting estimated rain rate and exact real rate. It shows initialization and fitted values of the different algorithms coefficients. After determining the parametrization coefficients, the rain rate was estimated with every algorithm using the generated radar data.

Figure 4 shows scatter plots of estimated rain rate obtained using (a) $R(Z)$, (b) $R\left(Z, Z_{\mathrm{dr}}\right)$, (c) $R\left(K_{\mathrm{dp}}\right)$, (d) $R\left(K_{\mathrm{dp}}, Z_{\mathrm{dr}}\right)$, and (e) $R\left(Z, K_{\mathrm{dp}}, Z_{\mathrm{dr}}\right)$ versus the exact rain rate computed from the known microphysical DSD properties. Figures $4 \mathrm{a}$ and $4 \mathrm{~b}$ show that $Z$-based algorithms present large deviations for rain rates over $50 \mathrm{~mm} \mathrm{~h}^{-1}$. This deviation is about $30 \%$ for the $R(Z)$ algorithm and about $25 \%$ for the $R\left(Z, Z_{\mathrm{dr}}\right)$ algorithm. However, they give good results for lower rain rates. On the other hand, from Fig. $4 \mathrm{c}$ and d, it is clear that $R\left(K_{\mathrm{dp}}\right)$ and $R\left(K_{\mathrm{dp}}, Z_{\mathrm{dr}}\right)$ present very large errors for small rain rates, though they provide good results for rain rates over $50 \mathrm{~mm} \mathrm{~h}^{-1}$ with errors under $15 \%$. Figure $4 \mathrm{e}$ presents the results corresponding to the proposed method. In this case, deviation errors are under $20 \%$ for all rain rates.

In order to further validate this result, a cumulative rain rate estimation test was performed. A rain event of two hours has been simulated. The rain rate was supposed to change every $15 \mathrm{~min}$, taken successively for the following values: 6 , $22,62,87,100,120,160$, and $200 \mathrm{~mm} \mathrm{~h}^{-1}$. A radar dwell time of 3 min has been assumed, which leads to 5 measurements for every rain rate value. Figure 5 shows the obtained results. We can deduce that all the existing algorithms give a slight overestimate relative to the exact rain accumulation. 
Table 2. Initialization and fitting coefficients for the different rain rate algorithms.

\begin{tabular}{|c|c|c|c|c|}
\hline Algorithm & Form & coefficient & Initialization value & Fitted value \\
\hline \multirow[t]{2}{*}{$R(Z)$} & \multirow[t]{2}{*}{$R=a Z^{b}$} & $\mathrm{a}$ & 0.038 & 0.035 \\
\hline & & $\mathrm{b}$ & 0.66 & 0.79 \\
\hline \multirow[t]{2}{*}{$R\left(K_{\mathrm{dp}}\right)$} & \multirow[t]{2}{*}{$R=a K_{\mathrm{dp}}^{b}$} & $\mathrm{a}$ & 44 & 46.18 \\
\hline & & $\mathrm{b}$ & 0.93 & 0.91 \\
\hline \multirow{3}{*}{$R\left(Z, Z_{\mathrm{dr}}\right)$} & \multirow{3}{*}{$R=a Z^{b} Z_{\mathrm{dr}}^{c}$} & $\mathrm{a}$ & 0.017 & 0.024 \\
\hline & & $\mathrm{b}$ & 1 & 0.95 \\
\hline & & $\mathrm{c}$ & -4.94 & -3.79 \\
\hline \multirow{3}{*}{$R\left(K_{\mathrm{dp}}, Z_{\mathrm{dr}}\right)$} & \multirow{3}{*}{$R=a K_{\mathrm{dp}}^{b} Z_{\mathrm{dr}}^{c}$} & $\mathrm{a}$ & 82,13 & 67.47 \\
\hline & & $\mathrm{b}$ & 1.16 & 0.97 \\
\hline & & $\mathrm{c}$ & -2.54 & -1.88 \\
\hline \multirow{4}{*}{$R\left(Z, K_{\mathrm{dp}}, Z_{\mathrm{dr}}\right)$} & \multirow{4}{*}{$R=a Z^{b} K-d p^{c} Z_{\mathrm{dr}}^{d}$} & $\mathrm{a}$ & 2.6 & 1.79 \\
\hline & & $\mathrm{b}$ & 0.56 & 0.52 \\
\hline & & $\mathrm{c}$ & 0.49 & 0.5 \\
\hline & & d & -3.89 & -1.76 \\
\hline
\end{tabular}

However, Fig. 5e shows that the proposed algorithm performs better in terms of the peak accumulation.

Moreover, the different given rain rate estimation algorithms were tested on radar data taken in light to moderate stratiform rainfalls with rain rates varying between 2 and $15 \mathrm{~mm} \mathrm{~h}^{-1}$. The data were collected by Matrosov et al. (2006), using experimental DSDs that were recorded by an impact Joss-Waldvogel disdrometer (JWD) deployed at the Boulder Atmospheric Observatory (BAO) during June of 2004. Thus, we got, $Z, Z_{\mathrm{dr}}$, and $K_{\mathrm{dp}}$ at S-band for experimental DSDs. The corresponding in-situ rain rates were acquired using the Colorado State University-University of Chicago Illinois State Water Survey (CSU-CHILL) radar. Two test sites were considered: the first one is located at BAO $(6.5 \mathrm{~km}$ from the NOAA radar and $54.5 \mathrm{~km}$ from CSU-CHILL), and the second one at the University of Colorado's Platteville (PLT) observatory $(27.8 \mathrm{~km}$ from the NOAA/ESRL radar and $30.4 \mathrm{~km}$ from CSU-CHILL). The considered coefficients for the rain rate expressions are those published in Bringi and Chandrasekar (2001). The numerical application in this case is given in Fig. 6a and b. The empirical results confirm the simulated ones. In fact, the rain rates estimated with the developed approach (based on the full set of polarimetric radar measurements) performs optimally.

The performance of the different rain rate estimation algorithms was quantitatively evaluated through the rainfall rate bias (computed as the absolute difference between the estimated rainfall rate and the closest measured one in time). Figure $6 \mathrm{c}$ and d illustrate, respectively, rainfall rate bias over BAO and PLT for the different algorithms. It can be seen that there is a good agreement with theory. In fact, over a rainfall rate of $8 \mathrm{~mm} \mathrm{~h}^{-1}$, the $K_{\mathrm{dp}}$-based rain rate estimation algorithm performs better than the $Z$-based ones. Thus, in Fig. $6 \mathrm{~d}$ the third measured sample, corresponding at around 22:40 UTC on 17 June over PLT, is an inflection point of the bias curve, as the three next measured samples have rainfall rates over $8 \mathrm{~mm} \mathrm{~h}^{-1}$. Moreover, the optimality of the $R\left(Z, K_{\mathrm{dp}}, Z_{\mathrm{dr}}\right)$ algorithm is confirmed, considering both cases of heavy and light rain. In fact, the bias red curves in Fig. $6 c$ and $d$ are always in the middle with respect to the bias green curves (computed for the $Z$ - based algorithms) and the blue ones (computed for the $K_{\mathrm{dp}}$-based algorithms).

\subsection{Estimation error analysis}

Now, let $\widehat{R}$ be the generic estimator from one of the five algorithms. Following Chandrasekar et al. (1993), the fluctuations of the errors in $\widehat{R}$ regarding the rain rate $R$ can be written as:

$\widehat{R}=R+\epsilon_{\mathrm{p}}+\epsilon_{\mathrm{m}}=R+\epsilon_{\mathrm{t}}$,

where $\epsilon_{\mathrm{p}}$ represents errors due to the parametric form of $\widehat{R}$, $\epsilon_{\mathrm{m}}$ represents errors due to the measurements, and $\epsilon_{\mathrm{t}}$ represents the total error. Simulations are performed in order to compute the normalized averages of $\epsilon_{\mathrm{t}}$, which represents the estimation algorithms biases as well as the normalized standard deviations $\sigma\left(\epsilon_{\mathrm{t}}\right) / R, \sigma\left(\epsilon_{\mathrm{p}}\right) / R$ and $\sigma\left(\epsilon_{\mathrm{m}}\right) / R$ for every algorithm. Monte Carlo simulations were performed in which the experience was repeated 150 times in order to obtain 150 rain rate estimate for every range gate. From these realizations, the biases and the standard deviations were computed. Normalization is performed in order to make obtained values more understandable since they can be interpreted as a percentile of errors. Firstly, different $\sigma\left(\epsilon_{\mathrm{t}}\right)$ are computed 


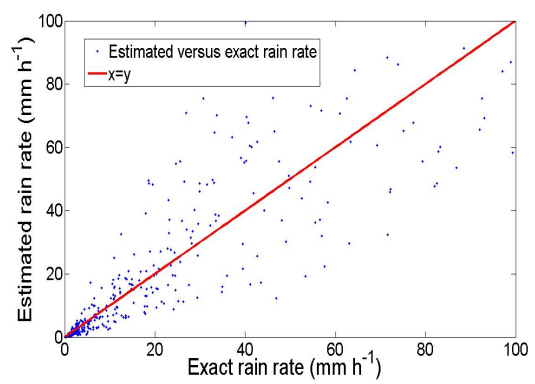

(a)

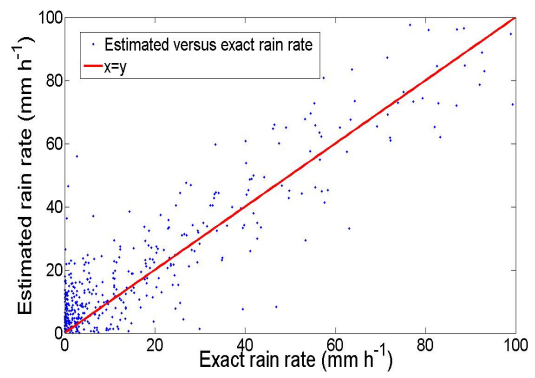

(c)

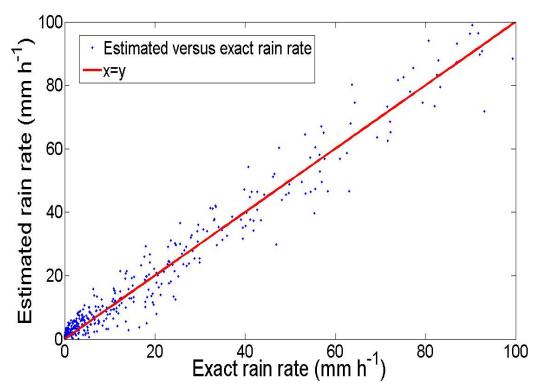

(e)

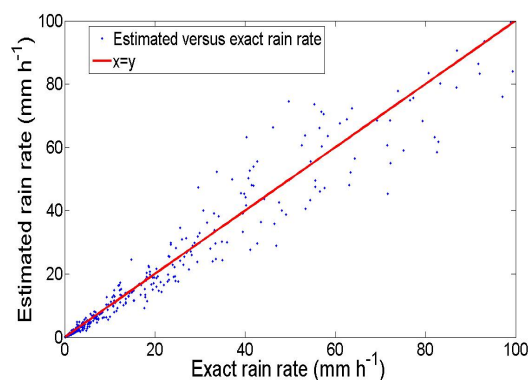

(b)

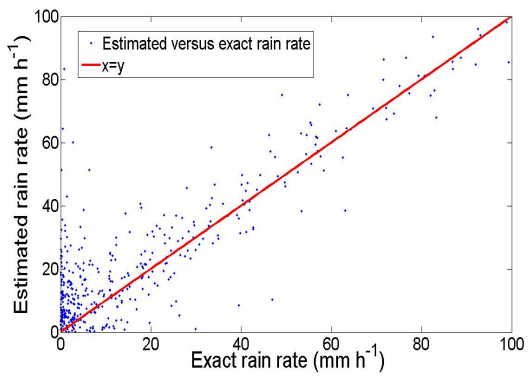

(d)

Fig. 4. Estimated versus exact rain rate. (a) $R(Z)$, (b) $R\left(Z, Z_{\mathrm{dr}}\right)$, (c) $R\left(K_{\mathrm{dp}}\right)$, (d) $R\left(K_{\mathrm{dp}}, Z_{\mathrm{dr}}\right)$, (e) $R\left(Z, K_{\mathrm{dp}}, Z_{\mathrm{dr}}\right)$.

from simulated real-like data. Then $\sigma\left(\epsilon_{\mathrm{p}}\right)$ are computed using simulated exact (only physically-based) data, and $\sigma\left(\epsilon_{\mathrm{m}}\right)$ are deduced from:

$\sigma\left(\epsilon_{\mathrm{m}}\right)=\sqrt{\sigma^{2}\left(\epsilon_{\mathrm{m}}\right)-\sigma^{2}\left(\epsilon_{\mathrm{p}}\right)}$.

Figure 7 shows the biases obtained for the different algorithms. As expected, the $K_{\mathrm{dp}}$-based algorithms present very high biases for low rain rates, and very low biases for high rain rates. However, the $Z$-based algorithms as well as the proposed one present acceptable results in both high and low rain rates.

In Bringi and Chandrasekar (2001), the expressions for relative bias error is given for the different existing rain rate estimation algorithms $\left(R(Z), R\left(Z, Z_{\mathrm{dr}}\right), R\left(K_{\mathrm{dp}}\right)\right.$, and $R\left(K_{\mathrm{dp}}, Z_{\mathrm{dr}}\right)$. In order to operate an inter-comparison with the Bringi formulas, the retrieval error for the adopted technique is developed. Using perturbation analysis and Eq. (27) we deduct the variance of $\epsilon_{\mathrm{m}}$ of $R\left(Z, K_{\mathrm{dp}}, Z_{\mathrm{dr}}\right)$ as,

$$
\frac{\operatorname{var}\left(\epsilon_{\mathrm{m}}\right)}{R^{2}}=b^{2}(x) \frac{\operatorname{var}(Z)}{Z^{2}}+c^{2}(x) \frac{\operatorname{var}\left(K_{\mathrm{dp}}\right)}{K_{\mathrm{dp}}^{2}}+d^{2}(x) \frac{\operatorname{var}\left(Z_{\mathrm{dr}}\right)}{Z_{\mathrm{dr}}^{2}}
$$

As $K_{\mathrm{dp}}$ is estimated over a path (say, $L=N \Delta r$ ), the evaluation of the relative bias error of the rain rate $\left(R\left(Z, K_{\mathrm{dp}}, Z_{\mathrm{dr}}\right)\right)$ have to be computed for a fixed path length. Thus, we considered the same numerical application as given by Bringi (Bringi and Chandrasekar (2001), in Sect. 8.3.3): the standard error in the measurement of reflectivity is 0.8 $\mathrm{dB}$, error in the measurement of $Z_{\mathrm{dr}}, L=3 \mathrm{~km}$ and $\Delta r=$ $0.15 \mathrm{~km}$. The variance of $Z$ is also reduced by a factor of $\sqrt{N}$. Figure 8 shows the obtained standard deviations, respectively, for (a) $R(Z)$, (b) $R\left(Z, Z_{\mathrm{dr}}\right)$, (c) $R\left(K_{\mathrm{dp}}\right)$, (d) $R\left(K_{\mathrm{dp}}, Z_{\mathrm{dr}}\right)$, and (e) $R\left(Z, K_{\mathrm{dp}}, Z_{\mathrm{dr}}\right)$. Again, these plots confirm the good behavior of the proposed method, since it provides an acceptable standard deviation for light rain rates. Also, it provides good standard deviations for heavy rain 

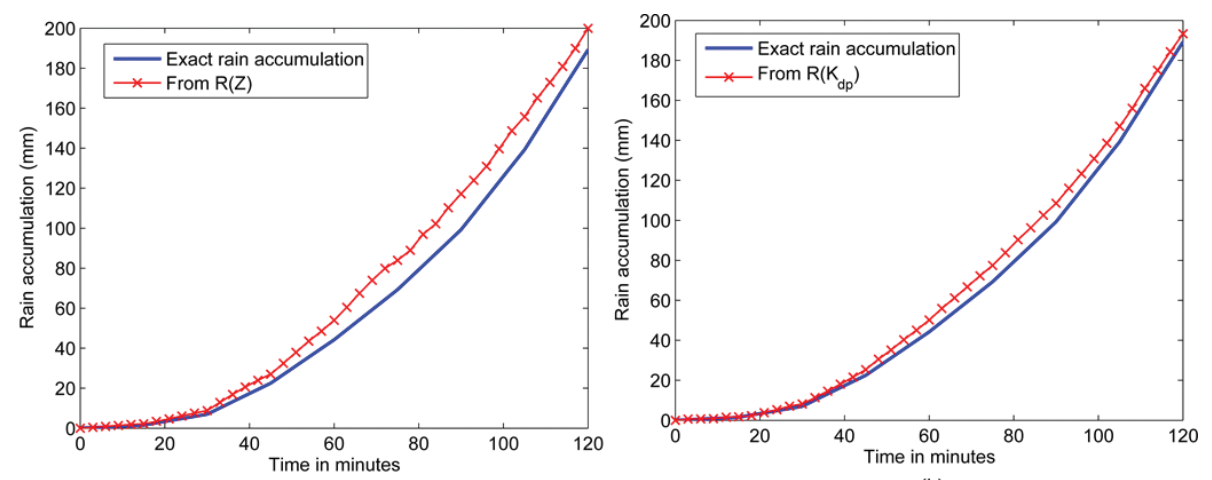

(a)
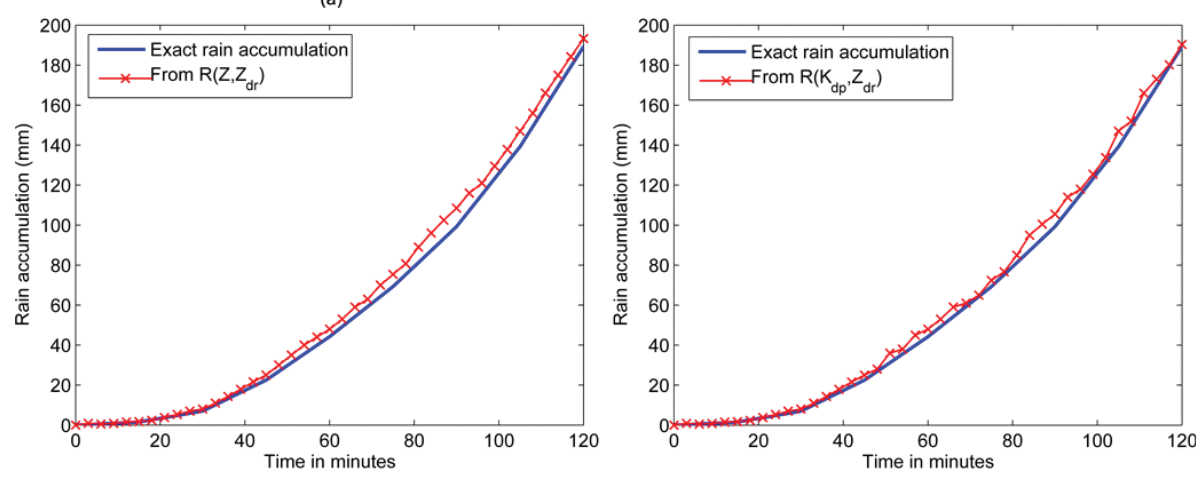

(c)

(d)

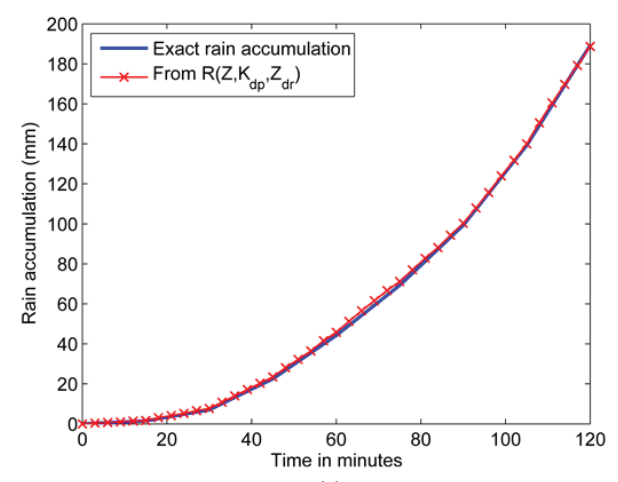

(e)

Fig. 5. Rain accumulation. (a) $R(Z)$, (b) $R\left(K_{\mathrm{dp}}\right)$, (c) $R\left(Z, Z_{\mathrm{dr}}\right)$, (d) $R\left(K_{\mathrm{dp}}, Z_{\mathrm{dr}}\right)$, (e) $R\left(Z, K_{\mathrm{dp}}, Z_{\mathrm{dr}}\right)$. Figure 7 Obtained biases for the physically-based rain rate estimation algorithm.

rates. So, the proposed method presents a very interesting tradeoff, allowing good rain rate estimates simultaneously for both light and heavy rain.

\section{Conclusions}

A rain rate estimation algorithm adapted for regions with light and heavy rain has been presented, assuming a well calibrated radar. It is based on $Z_{\mathrm{dr}}, Z$, and $K_{\mathrm{dp}}$ measurements. A "real-like" weather radar generator has also being introduced. This rain signals simulator is based on both statistical and physical modes. Thus, using the ergodicity principle, it makes possible the generation of "real-like" radar data starting from fixed physical properties of the drops. Simulations made with generated data show that the proposed one performs well for both light and heavy rain rates compared to other algorithms. These results were also validated through in-situ data. The data were collected by Matrosov et al. (2006), using experimental DSDs that were recorded by an impact Joss-Waldvogel disdrometer (JWD) deployed at the Boulder Atmospheric Observatory (BAO) during June of 2004. Thus, we got $Z, Z_{\mathrm{dr}}$, and $K_{\mathrm{dp}}$ at S-band for experimental DSDs. The corresponding in-situ rain rates were acquired using the Colorado State University-University of Chicago Illinois State Water Survey (CSU-CHILL) radar. Two test sites were considered: the first one is located at $\mathrm{BAO}(6.5 \mathrm{~km}$ 


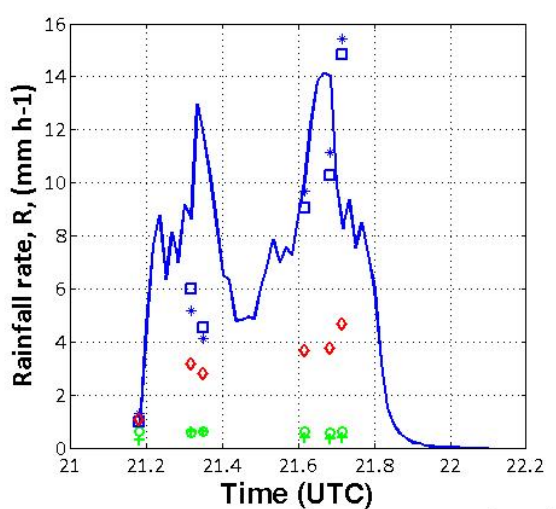

(a)

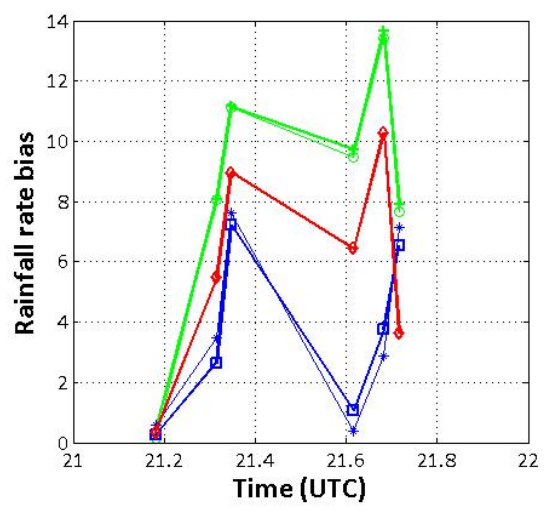

(C)

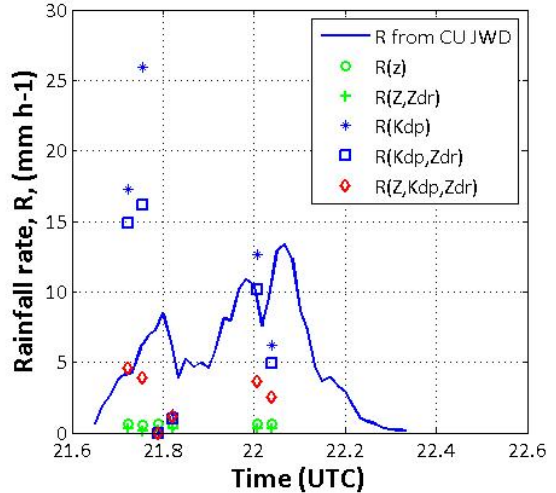

(b)

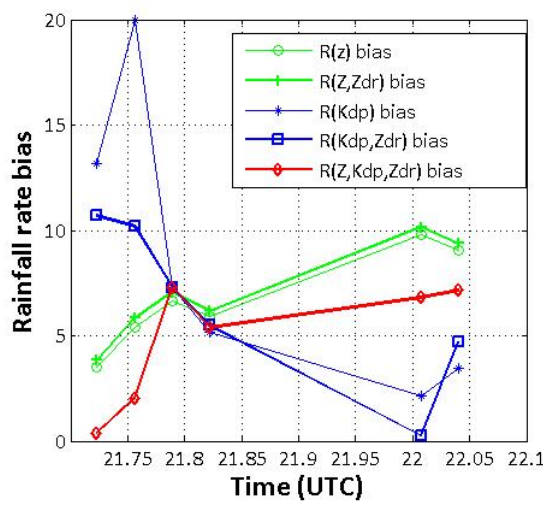

(d)

Fig. 6. Rain rates for light to moderate rainfalls over (a) BAO and (b) PLT on 17 June 2004 and, respectively, their corresponding rainfall rate bias (c) for BAO and (d) for PLT.

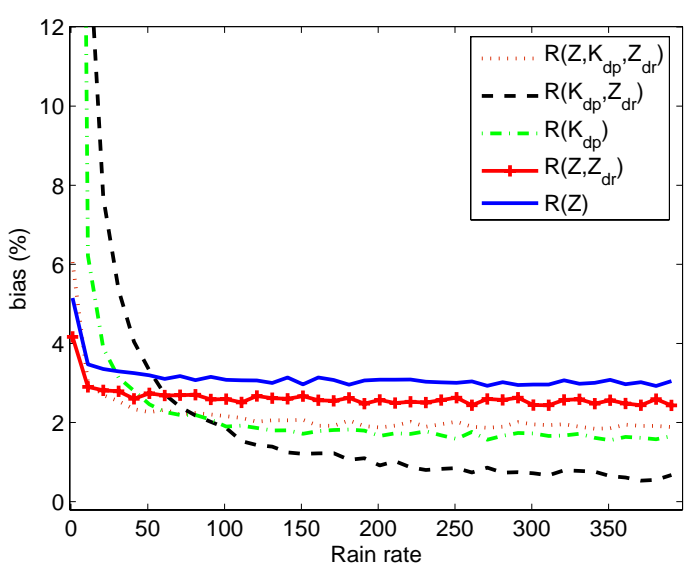

Fig. 7. The estimator bias versus the rain rate.

from the NOAA radar and $54.5 \mathrm{~km}$ from CSU-CHILL), and the the second one at the University of Colorado's Platteville (PLT) observatory (27.8 km from the NOAA/ESRL radar and $30.4 \mathrm{~km}$ from CSU-CHILL).

www.nat-hazards-earth-syst-sci.net/11/3067/2011/

\section{Appendix A}

\section{Covariance matrix elements computations}

The model supposes that $\psi$ and $\beta$ are independent and follow, respectively, $\mathcal{N}\left(\psi_{0}, \sigma_{\psi}\right)$ and $\mathcal{N}\left(\beta_{0}, \sigma_{\beta}\right)$. This means that the rain target verifies a reflection symmetry around the axis defined by the couple of angles $\left(\psi_{0}, \beta_{0}\right)$. In these conditions, the off-diagonal covariance matrix are zeros and the remaining elements are given by:

$$
\begin{aligned}
<\left|S_{\mathrm{hh}}\right|^{2}> & =<\left(A f_{\mathrm{a}}+B f_{\mathrm{b}}\right)\left(A f_{\mathrm{a}}+B f_{\mathrm{b}}\right)^{*}> \\
& =\overline{A^{2}}<\left|f_{\mathrm{a}}\right|^{2}>+\overline{A B}\left(<f_{\mathrm{a}} f_{\mathrm{b}}^{*}>+<f_{\mathrm{a}}^{*} f_{\mathrm{b}}>\right)+ \\
<\left|S_{\mathrm{vv}}\right|^{2}> & =<\left(\left.C f_{\mathrm{b}}\right|^{2}>\right. \\
& \left.=\overline{C^{2}}<\left|f_{\mathrm{a}}\right|^{2}>+\bar{C}\right)\left(C f_{\mathrm{a}}+D f_{\mathrm{b}}\right)^{*}> \\
& \overline{D^{2}}<\left|f_{\mathrm{b}}\right|^{2}> \\
<\left|S_{\mathrm{vh}}\right|^{2}> & =<\left(E\left(f_{\mathrm{b}}-f_{\mathrm{a}}\right)\right)\left(E\left(f_{\mathrm{b}}-f_{\mathrm{a}}\right)\right)^{*}> \\
& \left.=\overline{E^{2}}<\mid f_{\mathrm{b}}-f_{\mathrm{b}}>\right)+ \\
<S_{\mathrm{hh}} S_{\mathrm{vv}}^{*}> & =<\left(A f_{\mathrm{a}}+B f_{\mathrm{b}}\right)\left(C f_{\mathrm{a}}+D f_{\mathrm{b}}\right)^{*}> \\
= & \overline{A C}<\left|f_{\mathrm{a}}\right|^{2}>+\overline{A D}<f_{\mathrm{a}} f_{\mathrm{b}}^{*}>+\overline{B C}<f_{\mathrm{a}}^{*} f_{\mathrm{b}}>+ \\
& \overline{B D}<\left|f_{\mathrm{b}}\right|^{2}>
\end{aligned}
$$

where the $\langle\cdots\rangle$ represents the integration over drop size, and $\cdots$ represents the average over orientation. Remember 


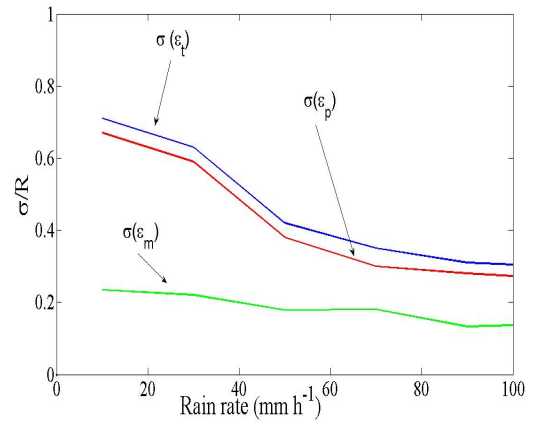

(a)

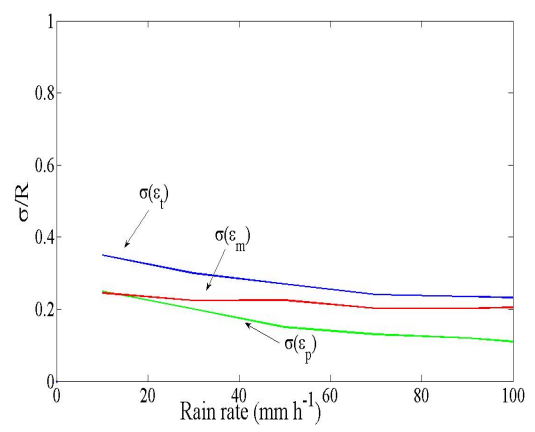

(c)

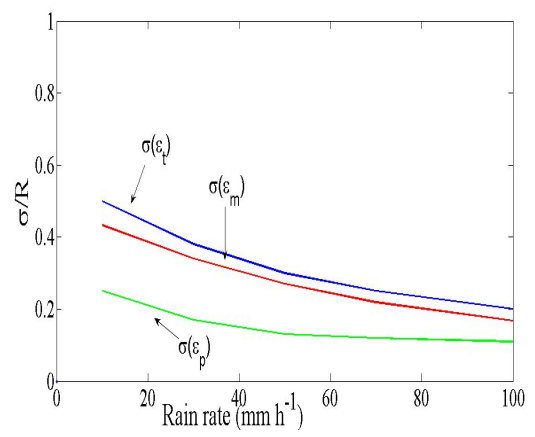

(e)

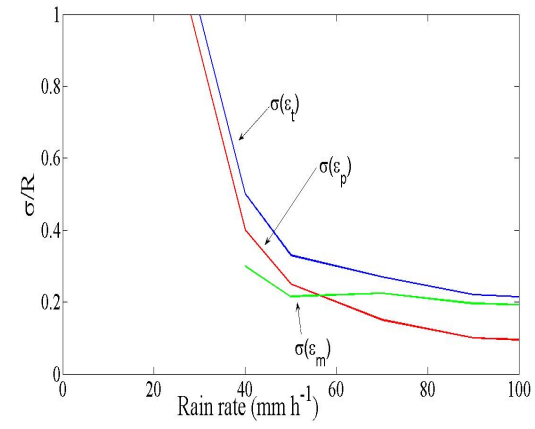

(b)

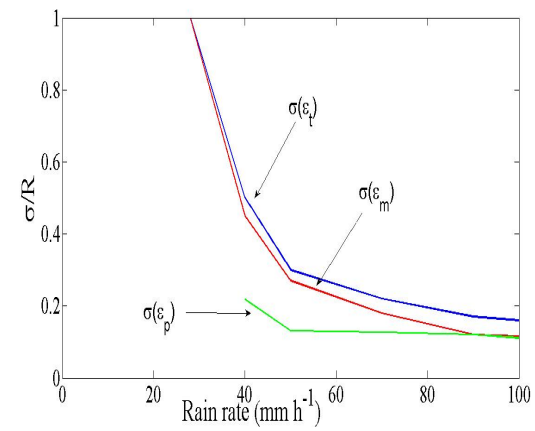

(d)

Fig. 8. Standard deviations, considered from Bringi and Chandrasekar (2001) (a) $R(Z)$, (b) $R\left(K_{\mathrm{dp}}\right)$, (c) $R\left(Z, Z_{\mathrm{dr}}\right)$, (d) $R\left(K_{\mathrm{dp}}, Z_{\mathrm{dr}}\right)$, and for the proposed algorithm (e) $R\left(Z, K_{\mathrm{dp}}, Z_{\mathrm{dr}}\right)$.

that it was assumed that orientation and particle size distributions are independents. The model supposes that $\psi$ and $\beta$ are independent and follow, respectively, $\mathcal{N}\left(\psi_{0}, \sigma_{\psi}\right)$ and $\mathcal{N}\left(\beta_{0}, \sigma_{\beta}\right)$. So, the values of $\overline{A^{2}}, \overline{A B}, \overline{A C}, \overline{A D}, \overline{B^{2}}, \overline{B C}$, $\overline{B D}, \overline{C^{2}}, \overline{C D}$, and $\overline{D^{2}}$ can be easily evaluated using the following relationships:

$$
\begin{aligned}
& \int_{-\infty}^{+\infty} \sin ^{2}(x) \frac{1}{\sqrt{2 \pi} \sigma_{x}} e^{\frac{-\left(x-x_{0}\right)^{2}}{2 \sigma_{x}^{2}}} d x=\frac{1}{2}\left(1-e^{\left.-2 \sigma_{x}^{2} \cos \left(2 x_{0}\right)\right)}\right. \\
& \int_{-\infty}^{+\infty} \cos ^{2}(x) \frac{1}{\sqrt{2 \pi} \sigma_{x}} e^{\frac{-\left(x-x_{0}\right)^{2}}{2 \sigma_{x}^{2}}} d x=\frac{1}{2}\left(1+e^{\left.-2 \sigma_{x}^{2} \cos \left(2 x_{0}\right)\right)}\right. \\
& \int_{-\infty}^{+\infty} \sin ^{4}(x) \frac{1}{\sqrt{2 \pi} \sigma_{x}} e^{\frac{-\left(x-x_{0}\right)^{2}}{2 \sigma_{x}^{2}}} d x=\frac{3}{8}+\frac{e^{8 \sigma_{x}^{2}} \cos \left(4 x_{0}\right)}{8}-\frac{e^{-2 \sigma_{x}^{2}} \cos \left(2 x_{0}\right)}{2} \\
& \int_{-\infty}^{+\infty} \cos ^{4}(x) \frac{1}{\sqrt{2 \pi} \sigma_{x}} e^{\frac{-\left(x-x_{0}\right)^{2}}{2 \sigma_{x}^{2}}} d x=\frac{3}{8}+\frac{e^{8 \sigma_{x}^{2}} \cos \left(4 x_{0}\right)}{8}+\frac{e^{-2 \sigma_{x}^{2}} \cos \left(2 x_{0}\right)}{2}
\end{aligned}
$$

If we suppose that:

$f_{\mathrm{a}}=\alpha_{1} D^{\beta_{1}}$
$f_{\mathrm{b}}=\alpha_{2} D^{\beta_{2}}$

we have:

$$
\begin{aligned}
<f_{a} f_{\mathrm{b}}> & =<\alpha_{1} D^{\beta 1} \alpha_{2} D^{\beta_{2}}> \\
& =\int_{0}^{+\infty} \alpha_{1} D^{\beta_{1}} \alpha_{2} D^{\beta_{2}} N_{0} D^{\mu} \mathrm{e}^{(-\Lambda D)} d D \\
& =N_{0} \alpha_{1} \alpha_{2} \int_{0}^{+\infty} D^{\left(\beta_{1}+\beta_{2}+\mu\right)} e^{(-\Lambda D)} d D \\
& =N_{0} \alpha_{1} \alpha_{2} \int_{0}^{+\infty} \Lambda^{-\left(\beta_{1}+\beta_{2}+\mu+1\right)} D^{\left(\beta_{1}+\beta_{2}+\mu\right)} e^{-D} d D \\
& =N_{0} \alpha_{1} \alpha_{2} \Lambda^{-\left(\beta_{1}+\beta_{2}+\mu+1\right)} \int_{0}^{+\infty} D^{\left(\beta_{1}+\beta_{2}+\mu+1\right)-1} \mathrm{e}^{-D} d D \\
& =N_{0} \alpha_{1} \alpha_{2} \Lambda^{-\left(\beta_{1}+\beta_{2}+\mu+1\right)} \Gamma\left(\beta_{1}+\beta_{2}+\mu+1\right)
\end{aligned}
$$

Given that $f_{\mathrm{a}}$ and $f_{\mathrm{b}}$ are real then it follows:

$$
\begin{aligned}
& <\left|f_{\mathrm{a}}\right|^{2}>=<f_{\mathrm{a}} f_{\mathrm{a}}>=N_{0} \alpha_{1}^{2} \Lambda^{-\left(2 \beta_{1}+\mu+1\right)} \Gamma\left(2 \beta_{1}+\mu+1\right) \\
& <\left|f_{\mathrm{b}}\right|^{2}>=<f_{\mathrm{b}} f_{\mathrm{b}}>=N_{0} \alpha_{2}^{2} \Lambda^{-\left(2 \beta_{2}+\mu+1\right)} \Gamma\left(2 \beta_{2}+\mu+1\right)
\end{aligned}
$$


Acknowledgements. The authors would like to thank Sergey Matrosov and Robert Cifelli for their time and their support in offering and explaining the data collected as part of the GPM pilot experiment. They also gratefully acknowledge the spanish Ministry of Science and Innovation for partially funding this research under project TEC2008-06736-C03-02.

Edited by: A. Mugnai

Reviewed by: two anonymous referees

\section{References}

Atlas, D.: Advances in radar meteorology, Advances in Geophysics, Landsberg and Mieghem, Eds, New York, Academic Press, 317-478, 1964.

Atlas, D.: Early foundations of the measurement of rainfall by radar, Radar in Meteorolog. D. Atlas, ED., American Meteorological society, Boston, J. Appl. Meteor., 86-97, 1990.

Atlas, D. and Ulbrich, C. W.: Path and area integrated rainfall measurement by microwave attenuation in $1-3 \mathrm{~cm}$ band, J. Appl. Meteor., 16, 1322-1331, 1977.

Atlas, D., Srivastava, R. C., and Shekhon, R. S.: Dopler radar characteristics of precipitation at vertical incidence, Rev. Geophys. Space Phys., 2, 1-35, 1973.

Beard, K. V. and Chuang, C. A new model for the equilibrium shape of raindrops, J. Atmos. Sci., 44, 1509-1524, 1987.

Beaver, J. and Bringi, V. N.: The application of the S-band polarimetric radar measurements to Ka band attenuation prediction, Proc. IEEE, 134, 431-437, 1997.

Bringi, V. N. and Chandrasekar, V.: Polarimetric Doppler Weather Radar, Principles and Applications, 1st Edn., Cambridge, UK, Cambridge Univ. Press, 2001.

Brandes, E. A., Ryzhkov, A. V. and Zrnic, D. S.: An evaluation of radar rainfall estimates from specific differential phase, J. Atmos. Oceanic Technol., 18, 363-375, 2001.

Bringi, V. N., Chandrasekar, V., and Xiao, R.: Raindrop axis ratio and size distributions in Florida rainshafts: An assessment of multiparameter radar algorithms, IEEE Trans. Geosci. Remote Sens., 36, 703-715, 1998.

Chandrasekar, V., Cooper, W. A., and Bringi, V. N.: Axis ratios and oscillation of raindrops, J. Atmos. Sci., 45, 1325-1333, 1988.

Chandrasekar, V., Gorgucci, E., and Scachilli, G.: Optimization of multiparameter radar estimates of rainfall, J. Appl. Meteor., 12, 1288-1293, 1993.

Doviak, R. J. and Zrnic, D. S.: Doppler Radar Weather Observations, Academic Press, New York, 1993.

Elmzoughi, A., Abdelfattah, R., Belhadj, Z., and Santalla Del Rio, V.: 2D weather radar data simulator using specific reflectivitie and phase measurements for the rain rate estimation algorithms validation, European Signal Processing Conference (EUSIPCO 2007), 1663-1666, 2007.
Giangrande, S. E. and Ryzhkov, A. V.: Estimation of Rainfall Based on the Results of Polarimetric Echo Classification., J. Appl. Meteorol. Climatol., 47, 2445-2462, 2008.

Green, A. W.: An approximation for the shapes of large raindrops, J. Appl. Meteor., 14, 1578-1583, 1975.

Guifu, Z., Vivekanandan, J., and Brandes, E.: A Method For Estimating Rain Rate And Drop Size Distribution From Polarimetric Radar Measurements IEEE Trans. Geosci. Remote Sens., 39, 830-841, 2001.

Laws, J. O. and Parsons, D. A.: The relationship of raindrop size to intensity, Trans. Amer. Geophys. Union, 24, 452-460, 1943.

Marquardt, D.: An Algorithm for Least Squares Estimation of Nonlinear Parameters, SIAM J. Appl. Math, 11, 431-441, 1963.

Marshall, J. S. and Palmer, W.: The distribution of raindrops with size, J. Meteor., 5, 165-166, 1948.

Matrosov, S. Y., Clark, A. K., Martner, B. E. and Tokay, A.: X-band polarimetric radar measurements of rainfall, J. Appl. Meteorol., 41, 941-952, 2002.

Matrosov, S. Y., Cifelli, R., Kennedy, P. C., Nesbitt, S. W., Rutledge, S. A., Bringi V. N., and Martner B. E.: A Comparative Study of Rainfall Retrievals Based on Specific Differential Phase Shifts at X- and S-Band Radar Frequencies, J. Atmos. Ocean. Tech., 23, 952-963, 2006.

McCormick, G. C. and Hendry, A.: Techniques for the determination of the polarization properties of precipitation, Radio Sci., 14, 1027-1040, 1979.

Oguchi, T.: Electromagnetic wave propagation and scattering in rain and other hyrdrometeors, IEEE. Proc., 71, 1029-1078, 1983.

Pruppacher, H. R. and Beard, K. V.: A wind tunnel investigation of the internal circulation and shape of water drops falling at terminal velocity in air, Meteor. Soc., 96, 247-256, 1970.

Ryzhkov, A. V., Giangrande, S. E., and Schuur, T. J.: Rainfall Estimation with a Polarimetric Prototype of WSR-88D, J. Appl. Meteorol., 44, 502-515, 2005.

Sachidananda, M. and Zrnic, D. S.: Rain rate estimates from differential polarization measurements, J. Atmos. Ocean. Technol., 4, 588-598, 1987.

Seliga, T. A. and Bringi, V. N.: Potential use of radar differential reflectivity measurements at orthogonal polarization for measuring precipitation, J. Appl. Meteor., 15, 69-76, 1976.

Tough, R. J. A., Blacknell, D., and Quegan, S.: A Statistical Description of Polarimetric Synthetic Aperture Radad Data, Proc. Roy. Soc., 449, 567-589, 1995.

Ulbrich, C. W.: Natural variations in the analytical form of raindrop size distributions, J. Climate Appl. Meteor., 22, 1764-1775, 1983. 